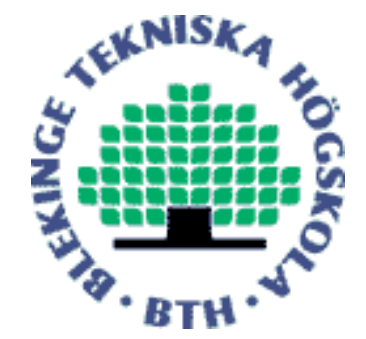

Copyright (C) 2009 IEEE.

Citation for the published paper:

Visual Attention Modelling for Subjective Image Quality Databases

Ulrich Engelke, Anthony Maeder, Hans-Jürgen Zepernick

International Workshop on Multimedia Signal Processing

2009 Rio de Janeiro

This material is posted here with permission of the IEEE. Such permission of the IEEE does not in any way imply IEEE endorsement of any of BTH's products or services Internal or personal use of this material is permitted. However, permission to reprint/republish this material for advertising or promotional purposes or for creating new collective works for resale or redistribution must be obtained from the IEEE by sending a blank email message to pubs-permissions@iee.org.

By choosing to view this document, you agree to all provisions of the copyright laws protecting it. 


\title{
Visual Attention Modelling for Subjective Image Quality Databases
}

\author{
Ulrich Engelke ${ }^{\# 1}$, Anthony Maeder ${ }^{* 2}$, Hans-Jürgen Zepernick \#3 \\ \# Blekinge Institute of Technology \\ PO Box 520, SE-372 25 Ronneby, Sweden \\ ${ }^{1}$ ulrichengelke@gmail.com \\ ${ }^{3}$ hans-jurgen.zepernick@bth.se \\ * University of Western Sydney \\ Locked Bag 1797, Penrith South DC, NSW 1797, Australia \\ 2 a.maeder@uws.edu.au
}

\begin{abstract}
The modelling of perceptual image quality metrics has experienced increased effort in recent years. In order to allow for model design, validation, and comparison, a number of subjective image quality databases has been made available to the research community. Most metrics that were designed using these databases assess the quality uniformly over the whole image, not taking into account stronger attention to salient regions of an image. In order to facilitate incorporation of visual attention (VA) into objective metric design we have conducted an eye tracking experiment. The experiment and its outcomes will be explained in detail in this paper. The actual gaze data recorded in the experiment is publicly available in order to facilitate and encourage VA modelling for image quality metrics.
\end{abstract}

\section{INTRODUCTION}

Perceptual image quality metrics aim on predicting quality as perceived by a human observer. As a basis for metric design, subjective experiments are usually conducted in which human observers have to rate the quality of a set of test images. The outcomes in terms of mean opinion scores (MOS) are considered as a ground truth to develop objective metrics. As such, image quality metrics build the transition from subjective assessment to an automated objective assessment. The ultimate goal is to replace traditional fidelity measures, such as the peak signal-to-noise ratio (PSNR), which are widely used but usually do not correlate well with human perception of quality.

The existing image quality metrics range from simple numerical metrics to complex metrics incorporating various properties of the human visual system (HVS). One important characteristic of the HVS, however, is often neglected in image quality metric design; visual attention (VA). This mechanism, in short, filters out redundant information and carries the focus to the salient regions and objects in our visual field. The level of attention can vary significantly and is influenced by many factors, such as the object's size, shape, and colour. The negligence of VA in image quality metric design is thought to be due to two reasons. Firstly, modelling VA is a highly difficult task and even though increased effort has been devoted towards VA modelling [1], [2], [3], reliable automatic detection of salient regions [4], [5], [6] is still an open research area. Secondly, and maybe more importantly, a subjective basis is needed for the development of the VA models similar to
MOS used for quality metric design. Such a VA ground truth is usually determined with eye tracking experiments [7] in which the gaze patterns of humans are recorded while observing a set of images. These experiments are time consuming though and usually require expensive equipment, such as the eye tracker hardware. They are, however, worth the effort since the incorporation of a VA model can significantly improve the prediction performance of image quality metrics [8].

There is a number of publicly available image quality databases (IQD), amongst which, some of the most widely used are the IVC [9], the LIVE [10], and the MICT [11] databases. These IQD allow the image quality research community to model and validate objective metrics and compare their performance, based on a common ground truth. The limitation, however, is that the IQD do not facilitate the modelling of VA. In order to bridge this gap we conducted an eye tracking experiment using the reference images from the above three databases. The eye tracking experiment will be presented in this paper along with the fixation density maps (FDM) [12] that we created to visualize the VA for all reference images. We have made the gaze patterns from the eye tracking experiment publicly available in the Visual Attention for Image Quality (VAIQ) database, in order to facilitate and encourage the incorporation of VA into image quality metric design.

The paper is organised as follows. Section II introduces the three IQD considered in this paper. Section III then explains in detail the eye tracking experiment that we conducted. In Section IV we discuss the creation of FDM and present the VA from the experiment for all images in the IQD. Finally, conclusions are drawn in Section V.

\section{Image Quality Databases}

In this section we will briefly introduce the three IQD which we considered in this paper. For the readers' convenience, a summary of the IQD is provided in Table I.

\section{A. IVC Database}

The IVC database [9] has been established by the Institut de Recherche en Communications et en Cybernétique (IRCCyN) in Nantes, France. Ten images of dimension $512 \times 512$ pixels 
TABLE I

OVERVIEW OF THE IVC, LIVE, AND MICT DATABASES

\begin{tabular}{l|ccc}
\hline \hline Database & IVC [9] & LIVE [10] & MICT [11] \\
\hline Number of reference images & 10 & 29 & 14 \\
Number of test images & 235 & 779 & 168 \\
Image widths & 512 & $480-768$ & $480-768$ \\
Image heights & 512 & $438-720$ & $488-720$ \\
Number of observers/image & 15 & $20-29$ & 16 \\
Assessment method & DSIS & SS & ACJ \\
\hline \hline
\end{tabular}

were selected to create a total of 235 test images using JPEG coding, JPEG2000 coding, locally adaptive resolution coding, and blurring. Fifteen observers then rated the quality of the test images as compared to the reference images using the double stimulus impairment scale (DSIS) [13].

\section{B. LIVE Database}

The LIVE database [10] is provided by the Laboratory for Image \& Video Engineering of the University of Texas at Austin, USA. Here, JPEG coding, JPEG2000 coding, Gaussian blur, white noise, and fast fading were applied to create a total of 779 test images from 29 reference images. The image widths are in the range 480-768 and the image heights in the range 438-720. Between 20-29 observers rated the quality of each image using a single stimulus (SS) assessment method.

\section{MICT Database}

The MICT database [11] has been made available by the Media Information and Communication Technology Laboratory of the University of Toyama, Japan. The MICT database contains 168 test images obtained from 14 reference images using JPEG and JPEG2000 source encoding. The image widths and heights are, respectively, in the ranges 480-768 and 488720. Sixteen observers rated the quality of the test images using the adjectival categorical judgement (ACJ) method [13].

\section{EyE TRACKING EXPERIMENT}

We conducted an eye tracking experiment at the University of Western Sydney, Australia. The experiment will be explained in detail in the following sections and an overview of the main aspects is provided in Table II.

\section{A. Participants}

A total of 15 people participated in the experiment who were all staff and students from the Campbelltown campus of the University of Western Sydney. The age ranged from 20 to 60 years with an average age of 42 years. Nine participants were male and six were female. Twelve participants stated that they were not involved with image analysis in their professional and private activities. Three participants were or had been earlier somewhat involved with image analysis; one with face recognition, one with astronomical imaging, and one with image restoration.
TABLE II

OVERVIEW OF THE EYE TRACKING EXPERIMENT.

\begin{tabular}{l|ll}
\hline \hline Participants & Number & 15 \\
& Male/female & $9 / 6$ \\
& Non-experts/experts & $12 / 3$ \\
& Occupation & University staff/students \\
& Average age & 42 \\
\hline Laboratory setup & Room & Low light conditions \\
& Viewing distance & approx. 60 cm \\
& Visual acuity testing & Snellen chart \\
\hline Monitor & Type & Samsung SyncMaster \\
& Size & $19 "$ \\
& Resolution & $1280 \times 1024$ \\
\hline Eye tracker & Type & EyeTech TM3 [14] \\
& Accuracy & approx. 1 deg visual angle \\
& Recording rate & approx. 40-45 GP/sec \\
& Mounting & Under the monitor \\
& Calibration & 16 point screen \\
\hline \hline Recorded data & Number of images & 42 \\
& Presentation order & Randomly \\
& Samples/person/image & approx. 480-540 \\
\hline & Time (images) & 12 sec \\
& Time (grey screen) & 3 sec \\
\hline
\end{tabular}

\section{B. Laboratory Setup}

The experiment was conducted in a laboratory with low light conditions. A Samsung SyncMaster monitor of size 19 " was used for image presentation. The screen resolution was $1280 \times 1024$. Any objects that may have distracted the observers' attention were removed from the area around the monitor. The eye tracker was installed under the screen and the participants were seated at a distance of approximately $60 \mathrm{~cm}$ from the screen. A Snellen chart was used to test the visual acuity of each participant prior to the session.

\section{Eye Tracker Hardware}

We used an EyeTech TM3 eye tracker [14] to record the gaze of the human observers. A photo of the TM3 eye tracker is shown in Fig. 1. The TM3 consists of an infrared camera and two infrared light sources, one on either side of the camera. The accuracy with which the gaze is recorded is approximately $1 \mathrm{deg}$ of visual angle. The eye tracker records gaze points (GP) at about $40-45 \mathrm{GP} / \mathrm{sec}$. A calibration of the TM3 for a particular person is done using a 16 point calibration screen.

\section{Stimuli Presentation}

We presented the reference images from the IVC, LIVE, and MICT databases. These databases contain a total of $10+29+$ $14=53$ reference images, however, 11 images have been used both in the LIVE and MICT databases. We used these images only once and as such, a total of 42 images was presented to the participants in random order. Each image was shown for 12 seconds with a mid-grey screen shown between images for 3 seconds. The mid-grey screen contained a fixation point in the center which we asked the participants to focus on. As such, 


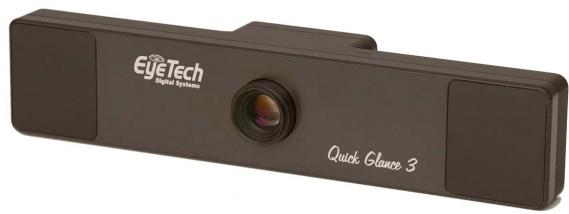

Fig. 1. EyeTech TM3 eye tracker [14] used in the experiment.

we assured that the observation of each image started at the same location. Given the presentation times and the number of images, the length of each session was about $10 \mathrm{~min}$.

\section{E. Recorded Data}

The TM3 tracks both eyes at the same time and records individual GP for each eye. An overall GP is then computed as the average between the two eyes. In addition, the TM3 records if an eye has been tracked in a particular time instance (eye status). If none of the two eyes could be tracked (for instance due to blinking) then the previous GP is recorded. These GP may be disregarded in a post-processing step since they contribute to attention where it might not actually be present. Given the recording rate of the eye tracker and the presentation time of each image, we recorded about 480-540 samples of each of the above data per person and image. This data is available in the VAIQ database.

\section{Visual Fixation PATterns}

Since the human retina is highly space variant in sampling and processing of visual information, an image usually cannot be apprehended with a single gaze. Thus, rapid eye movements (called saccades) are used to carry our focus to the salient regions of an image where fixations then allow for a more detailed observation. However, vision is suppressed during the saccades and as such, GP recorded during saccades provide only little information towards VA. Therefore, it is common practice to create visual fixation patterns (VFP) by clustering the GP of close spatial and temporal proximity and disregarding GP that were recorded during saccades. The creation of VFP is further motivated by the fact that GP visualized in an image are usually hard to comprehend due to the sheer amount of data. This is illustrated in Fig. 2 where the GP of all 15 participants are plotted within the same image.

In the next two sections we will present methodologies to create fixations from GP and also to visualize the resulting VFP. It should be noted that these methodologies are considered as a guide for prospective users of the VAIQ database who are not so familiar with VA modelling, rather than an exhaustive discussion on the creation and visualization of VFP.

\section{A. Creation of Visual Fixation Patterns}

A pseudo code for the creation of VFP from GP is provided in Alg. 1. Here, the $G P$ for a particular viewer and image are scanned in sequential order. The basic idea then is to assign the $G P$ to clusters $\mathcal{C}_{j}$ according to a pre-defined threshold $\tau_{\text {clus }}$. For this reason, the mean $\mu(j)$ is computed for all $G P$ in the current cluster including the new $G P(i)$ at a particular time instance $i$. If the distance of $G P(i)$ to the mean $\mu(j)$ is below

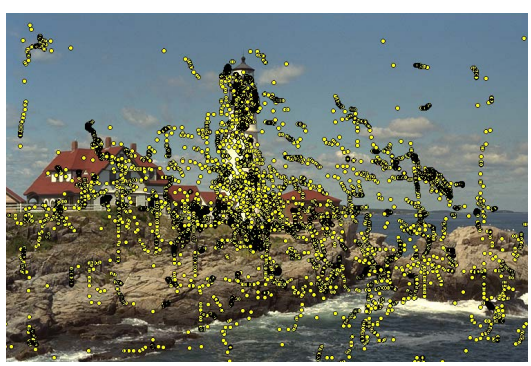

Fig. 2. Visualization of the GP for all 15 participants.

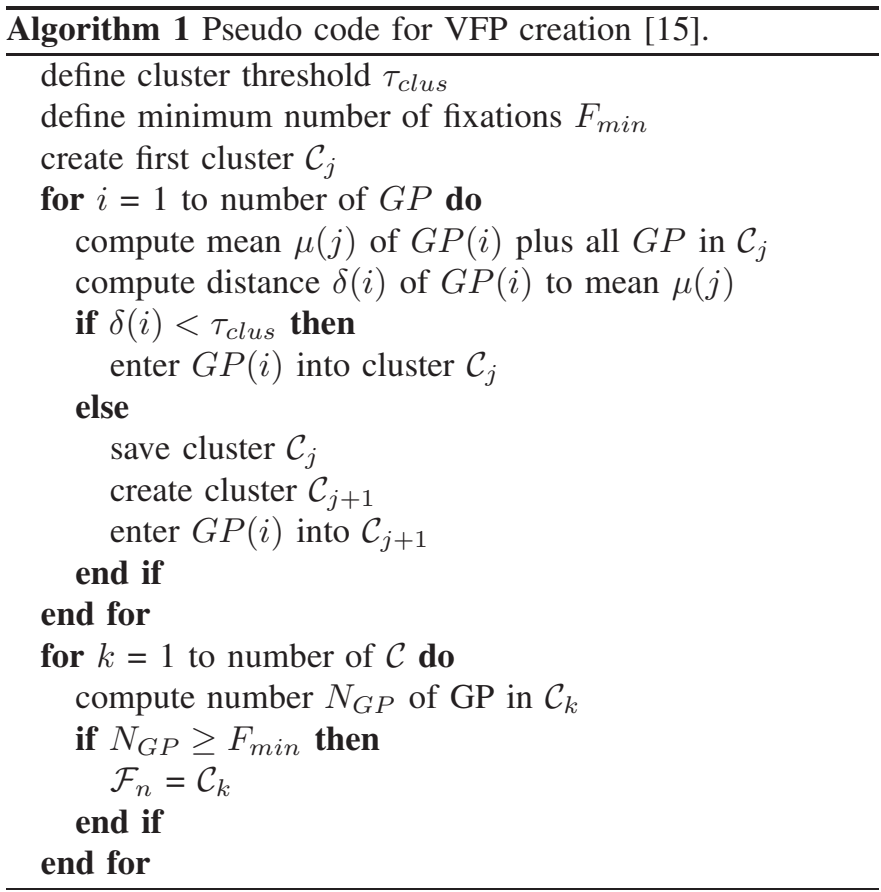

the threshold $\tau_{\text {clus }}$, then $G P(i)$ is added to the current cluster $\mathcal{C}_{j}$. If the distance is above the threshold, the current cluster $\mathcal{C}_{j}$ is saved and $G P(i)$ is added to the next cluster $\mathcal{C}_{j+1}$. After the clustering process, each cluster is considered to be a fixation $\mathcal{F}_{n}$ if it contains at least a pre-defined number, $F_{\text {min }}$, of GP. In our case we found that the algorithm performed well with $\tau_{\text {clus }}=20$ and $F_{\text {min }}=4$.

\section{B. Visualization Through Fixation Density Maps}

Fixation density maps (FDM) are an elegant way of visualizing the VFP. The pseudo code for the creation of FDM is given in Alg. 2. Here, we first initialize the FDM, $I_{F D M}$, and enter the fixations by means of single-pixel peaks. The amplitude of the peaks is in correspondence with the fixation lengths. The FDM is then convolved with a Gaussian filter kernel $\phi_{G}$, as illustrated in Fig. 7, to obtain $I_{F D M, \phi}$. We chose maximum filter dimensions of $x_{\max }=y_{\max }=70$ and a standard deviation of $\sigma=x_{\max } / 2=35$. The part above the grey threshold constitutes the area of the filter kernel that covers the corresponding pixels in the image which are processed with high acuity by the fovea. This threshold assumes a size of the fovea of 2 deg of visual angle and further 


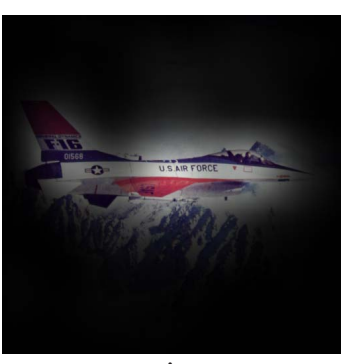

avion

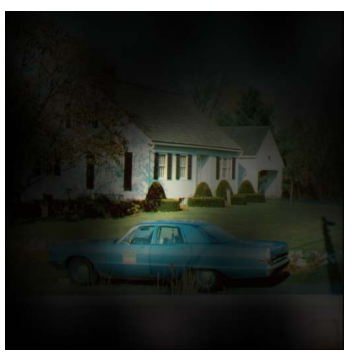

house

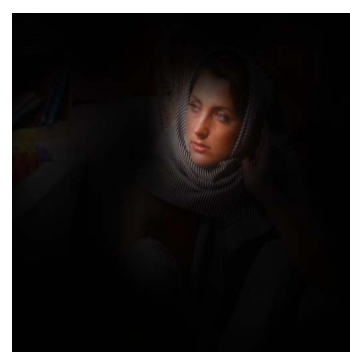

barba

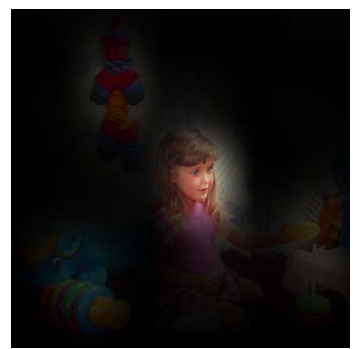

isabe

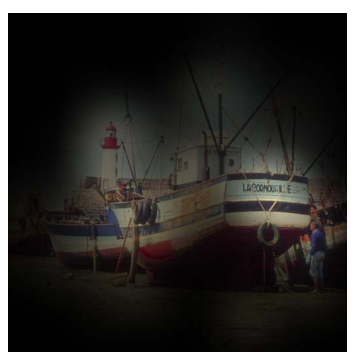

boats

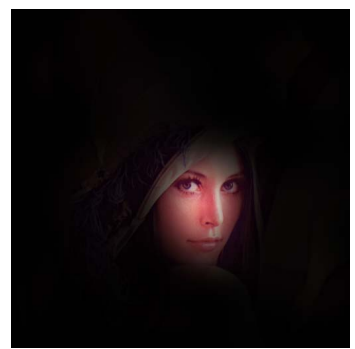

lenat

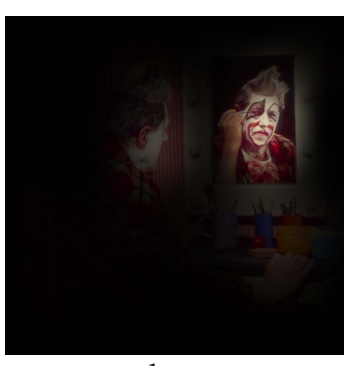

clown

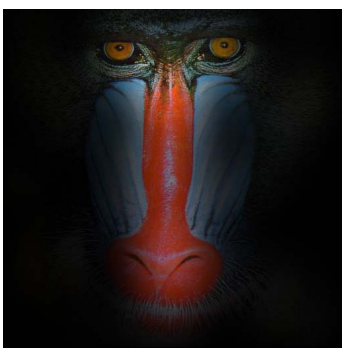

mandr

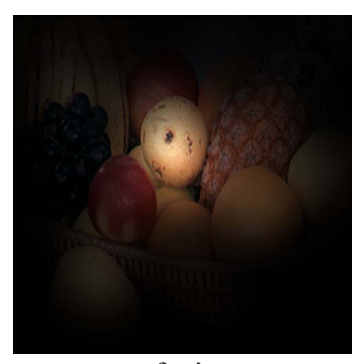

fruit

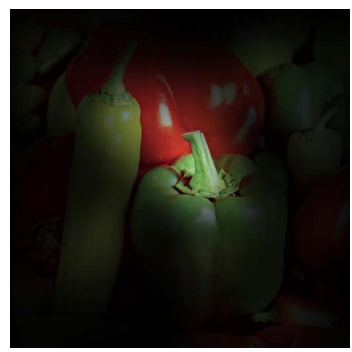

pimen

Fig. 3. Visualisation of VA with FDM for images from the IVC [9] database.

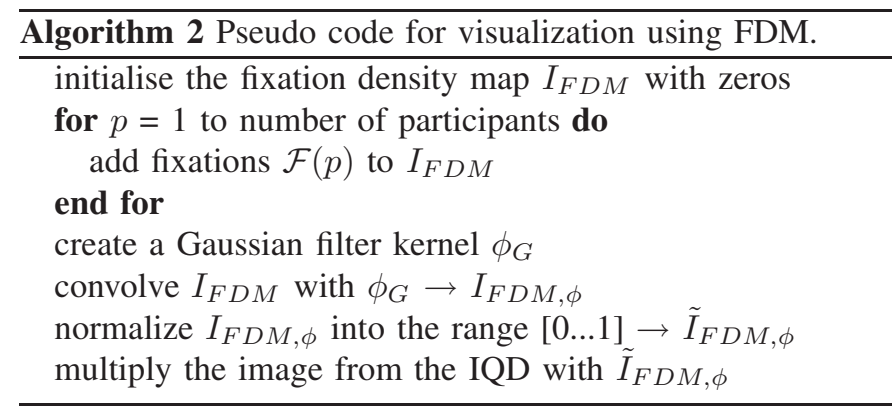

depends on the size of the presented image on the screen and the viewing distance. Finally, $I_{F D M, \phi}$ is normalized to a range of $[0 \ldots 1]$ and then multiplied pixel-by-pixel with the image from the IQD. As such, salient regions receive more brightness compared to the remainder of the image. The FDM for all 42 images are shown in Fig. 3-6. The labels under each image indicate the original names from the respective databases.

\section{Conclusions}

We have introduced the VAIQ database which we established based on the outcomes of an eye tracking experiment involving 15 human observers. The database facilitates the incorporation of VA models into image quality metrics that are designed based on the IVC, LIVE, and MICT databases. For those readers that are not so familiar with VA modelling, we have also provided guidelines for the creation and visualization of VFP. The VAIQ database is freely available to the research community. To obtain access to the VAIQ database please send an email to the lead author of this paper.

\section{ACKNOWLEDGEMENT}

The authors would like to thank all participants from the eye tracking experiment and Dr. Clinton Fookes for his assistance with the VFP algorithm.

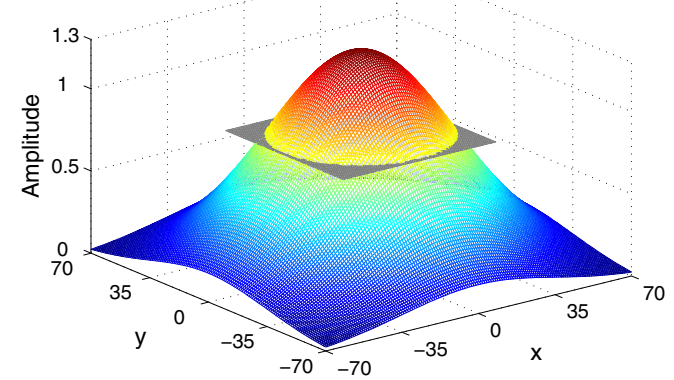

Fig. 7. Gaussian filter kernel with $\sigma=35$.

\section{REFERENCES}

[1] A. M. Treisman and G. Gelade, "A feature-integration theory of attention," Cognitive Psychology, vol. 12, no. 1, pp. 97-136, Jan. 1980.

[2] J. M. Wolfe, K. R. Cave, and S. L. Franzel, "Guided search: An alternative to the feature integration model for visual search," Journal of Experimental Psychology: Human Perception and Performance, vol. 15, no. 3, pp. 419-433, Aug. 1989

[3] L. Itti, C. Koch, and E. Niebur, "A model of saliency-based visual attention for rapid scene analysis," IEEE Trans. on Pattern Analysis and Machine Intelligence, vol. 20, no. 11, pp. 1254-1259, Nov. 1998.

[4] C. M. Privitera and L. W. Stark, "Algorithms for defining visual regionsof-interest: Comparison with eye fixations," IEEE Trans. on Pattern Analysis and Machine Intelligence, 2000.

[5] W. Osberger and A. M. Rohaly, "Automatic detection of regions of interest in complex video sequences," in Proc. of IS\&T/SPIE Human Vision and Electronic Imaging VI, vol. 4299, Jan. 2001, pp. 361-372.

[6] A. J. Maeder, "The image importance approach to human vision based image quality characterization," Pattern Recognition Letters, vol. 26 , no. 3, pp. 347-354, Feb. 2005

[7] A. L. Yarbus, Eye Movements and Vision. Plenum, 1967.

[8] U. Engelke and H.-J. Zepernick, "Optimal region-of-interest based visual quality assessment," in Proc. of IS\&T/SPIE Human Vision and Electronic Imaging XIV, vol. 7240, Jan. 2009.

[9] P. L. Callet and F. Autrusseau, "Subjective quality assessment IRCCyN/IVC database," http://www.irccyn.ec-nantes.fr/ivcdb/, 2005.

[10] H. R. Sheikh, Z. Wang, L. Cormack, and A. C. Bovik, "LIVE image quality assessment database release 2," http://live.ece.utexas.edu/research/quality, 2005. 


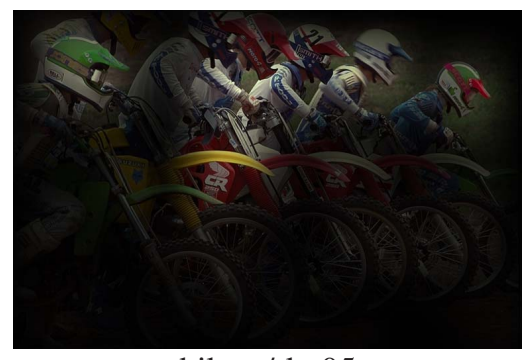

bikes / kp05

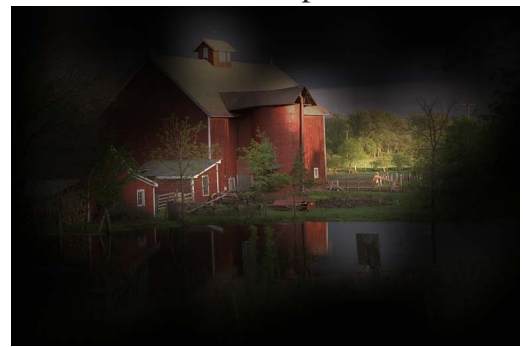

house / kp22

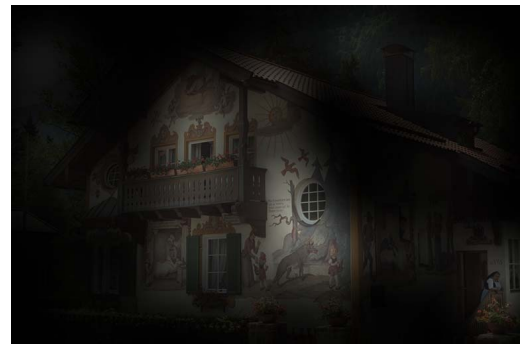

paintedhouse / kp24

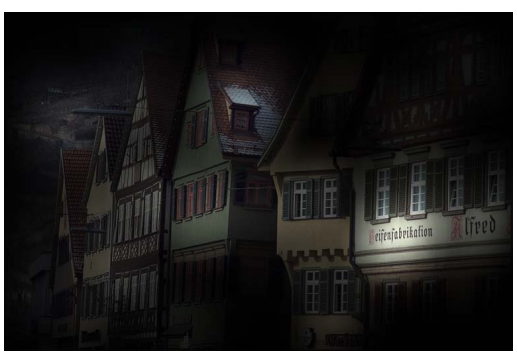

buildings / kp08

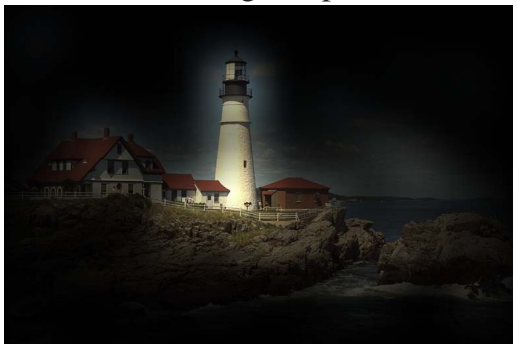

lighthouse2 / kp21

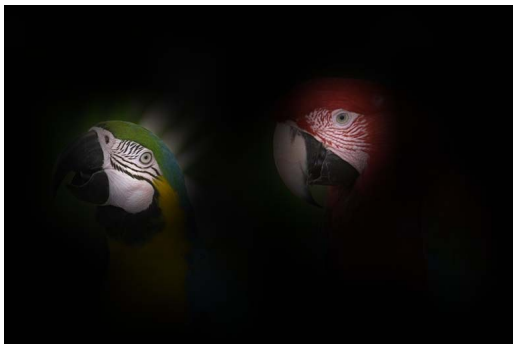

parrots / kp23

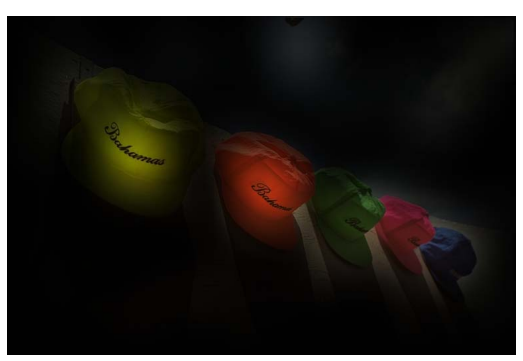

caps / kp03

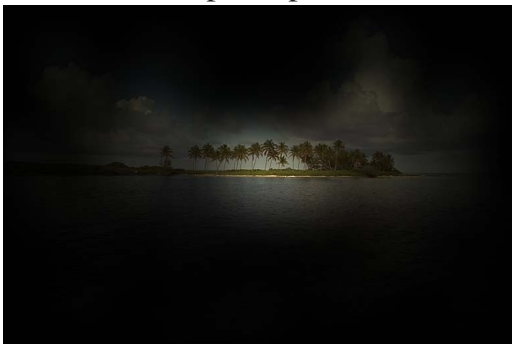

ocean / kp16

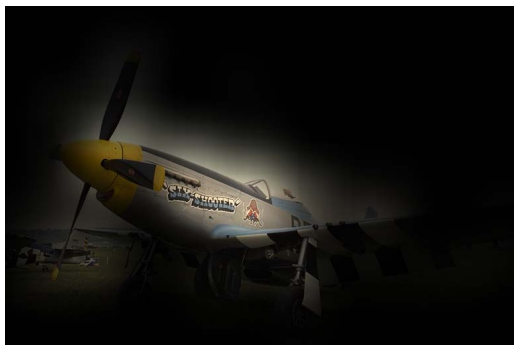

plane / kp20

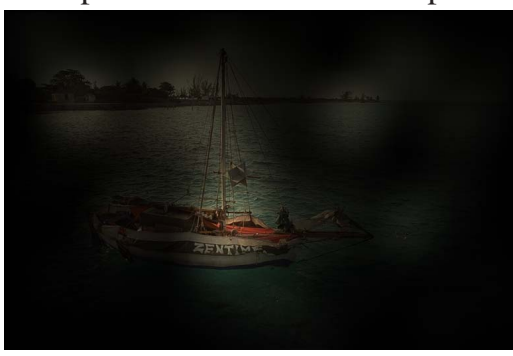

sailing1 / kp07

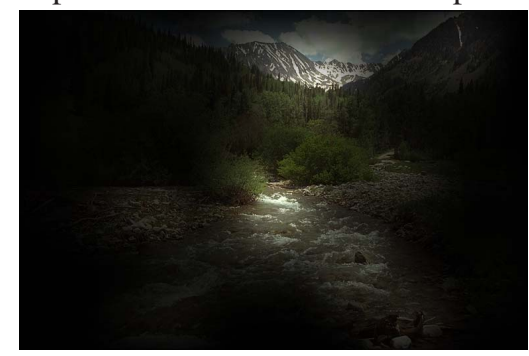

stream / kp13

Fig. 4. Visualisation of VA with FDM for images contained in both the LIVE [10] and the MICT [11] database (left label: LIVE; right label: MICT).

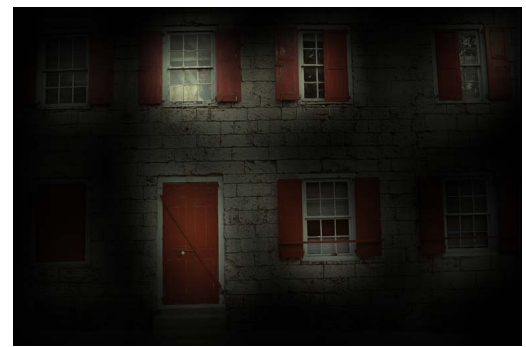

kp01

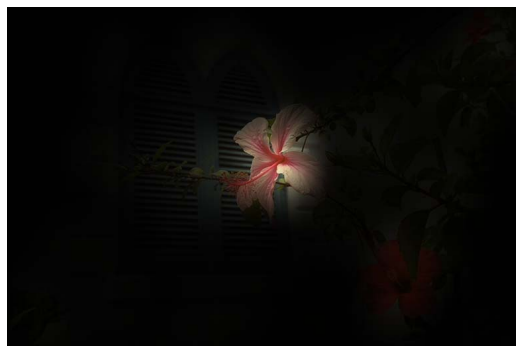

kp07

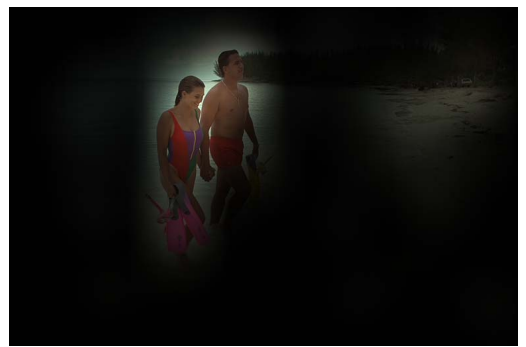

$\mathrm{kp} 12$

Fig. 5. Visualisation of VA with FDM for images exclusively from the MICT [11] database.

[11] Z. M. P. Sazzad, Y. Kawayoke, and Y. Horita, "Image quality evaluation database," http://mict.eng.u-toyama.ac.jp/database_toyama, 2000.

[12] O. L. Meur, P. L. Callet, D. Barba, and D. Thoreau, "A coherent computational approach to model bottom-up visual attention," IEEE Trans. on Pattern Analysis and Machine Intelligence, vol. 28, no. 5, pp. 802-817, May 2006.

[13] International Telecommunication Union, "Methodology for the sub- jective assessment of the quality of television pictures," ITU-R, Rec. BT.500-11, 2002

[14] EyeTech Digital Systems, "TM3 eye tracker," http://www.eyetechds.com/, 2009.

[15] C. Fookes, A. Maeder, S. Sridharan, and G. Mamic, "Gaze based personal identification," in Behavioral Biometrics for Human Identification: Intelligent Applications. IGI Global, 2009. 


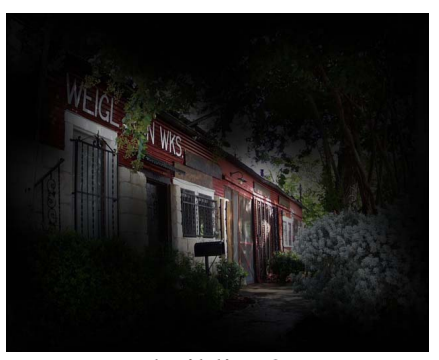

building2

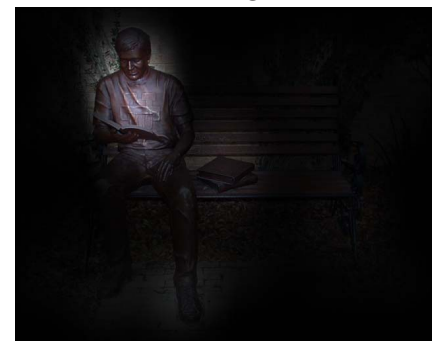

studentsculpture

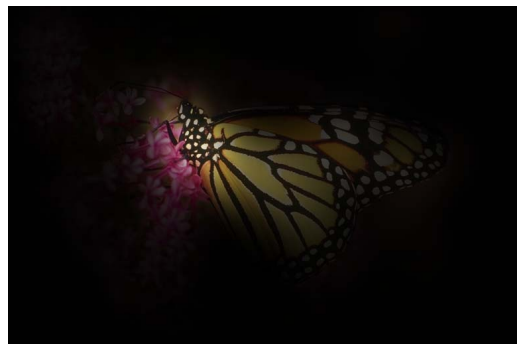

monarch

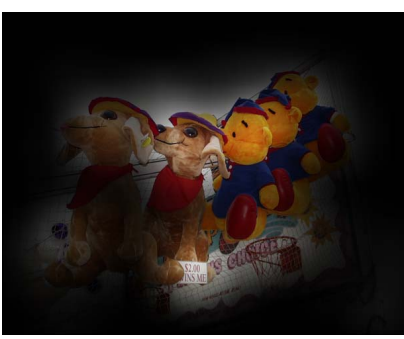

carnivaldolls

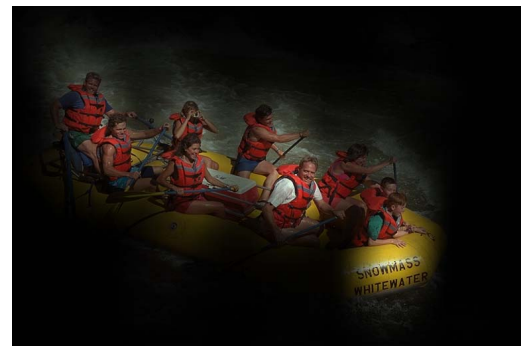

rapids

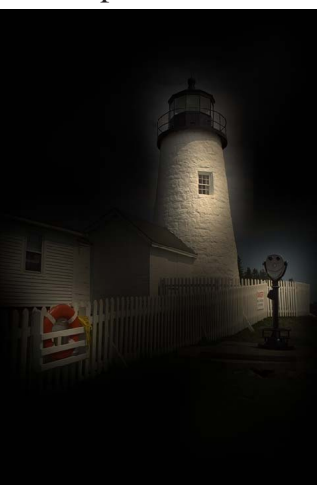

lighthouse

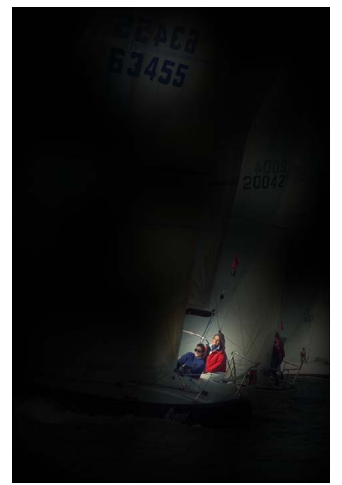

sailing3 churchandcapitol

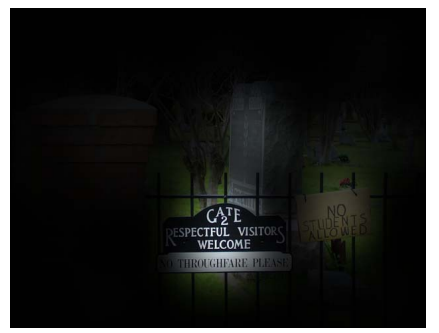

cemetry

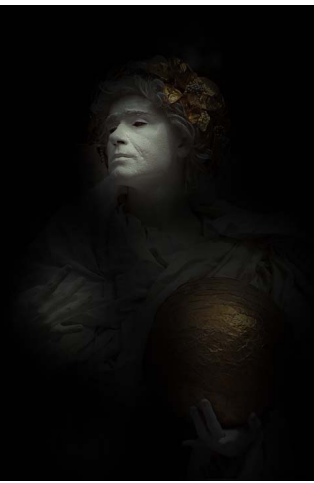

statue

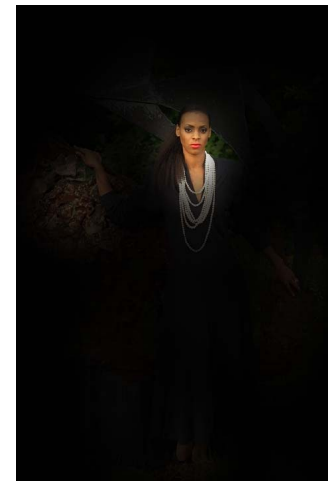

woman

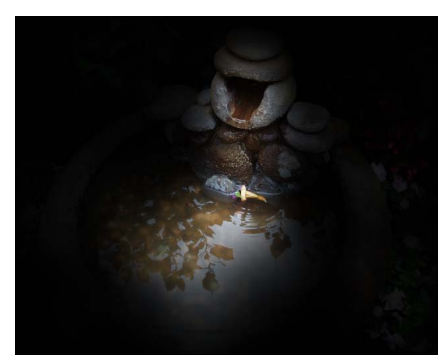

coinsinfountain

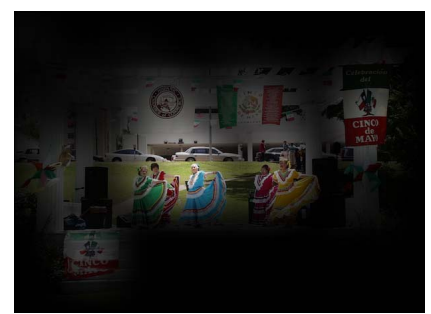

dancers

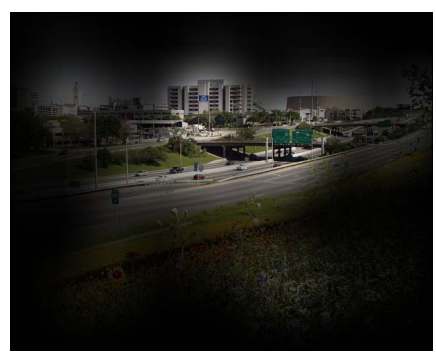

flowersonih35

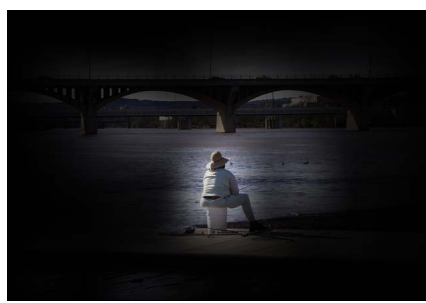

manfishing

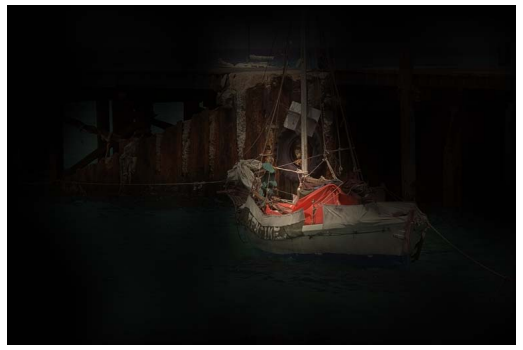

sailing4

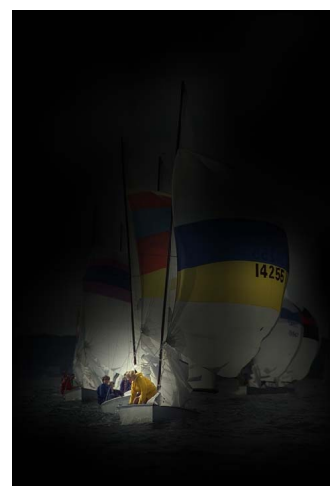

sailing2

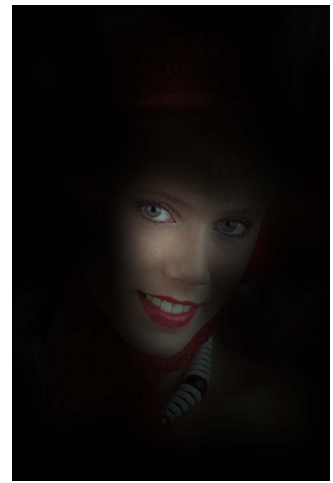

womanhat

Fig. 6. Visualisation of VA with FDM for images exclusively from the LIVE [10] database. 